\title{
15 mA Bidirectional Laser Triggering in Two-Terminal Microdevices Based on Vanadium Dioxide Thin Films
}

\author{
Eung-Soo Kim, Songhyun Jo ${ }^{1}$, Kyongsoo Park ${ }^{1}$, \\ Young-Suk Kim ${ }^{1}$ and Yong Wook Lee ${ }^{1,2, *}$ \\ Division of Digital Media Engineering, Busan University of Foreign Studies, \\ 65 Geumsaem-ro 485 beon-gil, Geumjeong-gu, Busan 609-815, Korea \\ 'Interdisciplinary Program of Biomedical Mechanical \& Electrical Engineering, \\ Pukyong National University, 45 Yongso-ro, Nam-gu, Busan 608-737, Korea \\ ${ }^{2}$ School of Electrical Engineering, Pukyong National University, \\ 45 Yongso-ro, Nam-gu, Busan 608-737, Korea
}

(Received July 14, 2014; accepted December 8, 2014)

Key words: vanadium dioxide, thin film, phase transition, microdevice, photoexcitation

By using an infrared laser diode with an oscillating wavelength of $1550 \mathrm{~nm}$, we demonstrated $15 \mathrm{~mA}$ bidirectional laser triggering with an amplitude switching ratio of 78.9 in a two-terminal microdevice based on a vanadium dioxide $\left(\mathrm{VO}_{2}\right)$ thin film, which was DC-biased at $3.35 \mathrm{~V}$. The $\mathrm{VO}_{2}$-based device has only one conducting layer, i.e., one $\mathrm{VO}_{2}$ patch, which facilitates the use of the triggering laser with a reduced spot diameter. The reduced beam spot can increase the maximum on-state current at the same illumination power, resulting in the improvement of the amplitude switching ratio. A specific bias voltage range enabling the bidirectional laser triggering was experimentally determined from the current-voltage characteristics of the $\mathrm{VO}_{2}$-based device with an electrode separation of $10 \mu \mathrm{m}$ and a current channel width of $30 \mu \mathrm{m}$, which was measured in a current-controlled mode. In a closed-loop circuit constructed by serially connecting a standard resistor, a DC voltage source, and the device, the transient responses of light-triggered currents were also observed for response time analysis.

\section{Introduction}

Vanadium dioxide $\left(\mathrm{VO}_{2}\right)$ is a representative strongly correlated material showing an insulator-to-metal or metal-to-insulator phase transition (PT) induced by external stimuli such as temperature, ${ }^{(1)}$ pressure, ${ }^{(2)}$ and light. ${ }^{(3)}$ This PT property of $\mathrm{VO}_{2}$ causes highly nonlinear current-voltage $(I-V)$ behavior in two-terminal devices based on $\mathrm{VO}_{2}$

*Corresponding author: e-mail: yongwook@pknu.ac.kr 
thin films, resulting in abrupt jumps of electrical currents. ${ }^{(4)}$ The threshold voltage of the device, after which a current jump occurs triggering the PT of $\mathrm{VO}_{2}$, is changed by illuminating the $\mathrm{VO}_{2}$ film with an infrared laser, ${ }^{(5,6)}$ and this laser-assisted switching is a basic function for the application of $\mathrm{VO}_{2}$-based devices to laser-triggered switches. In previous works, the maximum on-state current of the laser-triggered $\mathrm{VO}_{2}$ device was $\sim 3.9 \mathrm{~mA}$ with an amplitude ratio of less than 4 between switched variables (on- and off-state currents). ${ }^{(5)}$ Recently, $10 \mathrm{~mA}$ bidirectional laser triggering has been realized with an amplitude switching ratio of $\sim 68.2$ by incorporating $\mathrm{VO}_{2}$ devices with parallel conducting layers. ${ }^{(7)}$ For $\mathrm{VO}_{2}$-based devices to be considered for the practical application of optically gated switches such as light-triggered thyristors, numerous device parameters including the maximum on-state current, the amplitude switching ratio, and the offstate current should be significantly improved. In the optical triggering of $\mathrm{VO}_{2}$ devices, efforts for the enhancement of these parameters have been relatively insufficient, and most studies focused on the modulation of the optical properties of $\mathrm{VO}_{2}$ thin films, such as the refractive index and the transmittance. ${ }^{(8-13)}$ Some works on electrical switching devices based on $\mathrm{VO}_{2}$ thin films were reported by utilizing electrical pulses applied to two-terminal $\mathrm{VO}_{2}$-based devices. ${ }^{(14,15)}$

In this study, we demonstrated $15 \mathrm{~mA}$ bidirectional laser triggering in a two-terminal microdevice based on a $\mathrm{VO}_{2}$ thin film, fabricated by a sol-gel method, by using an approximately $1550 \mathrm{~nm}$ infrared laser diode (LD). The bidirectional laser triggering indicates that the forward or reverse $\mathrm{PT}$ of $\mathrm{VO}_{2}$ is triggered in accordance with the switched state (on- or off-state) of the LD. The bidirectional laser triggering can be regarded as optically controlled threshold switching realized by photoassisted negative differential resistance (NDR) reduction of $\mathrm{VO}_{2} \cdot{ }^{(7,16)}$ Here, unlike the devices used in the previous study, ${ }^{(7)}$ the $\mathrm{VO}_{2}$ device has only one conducting layer, i.e., one $\mathrm{VO}_{2}$ patch, which facilitates the use of an illumination laser with a reduced beam diameter. The reduced beam spot can increase the maximum on-state current at the same illumination power, resulting in the improvement of the amplitude switching ratio. In the following section, the experimental setup for inducing the laser triggering in the $\mathrm{VO}_{2}$ device will be explained including the fabrication of the device and the closed-loop circuit for measuring the transient response of laser-triggered current. The $I-V$ characteristics of the device triggered by the LD will be analyzed. Then, the transient response of the lasertriggered device will be investigated.

\section{Experimental Preparation}

Figure 1(a) shows the experimental setup for $15 \mathrm{~mA}$ laser triggering in a two-terminal $\mathrm{VO}_{2}$-based microdevice. An inset of Fig. 1(a) shows the plane-view optical microscopy image of the fabricated device $(L=10 \mu \mathrm{m}$ and $W=30 \mu \mathrm{m})$. The cross-sectional-view schematic diagram of the fabricated device is shown on the lower left side of Fig. 1(a). $\mathrm{VO}_{2}$ thin films whose average thickness was $\sim 100 \mathrm{~nm}$ were grown on sapphire $\left(\mathrm{Al}_{2} \mathrm{O}_{3}\right)$ substrates by sol-gel deposition. ${ }^{(17)}$ The sol-gel deposition method is composed of a series of spin coating and subsequent annealing processes. The $0.12 \mathrm{M}$ solution for the spin coating process was prepared by synthesizing vanadium triisopropoxide, 


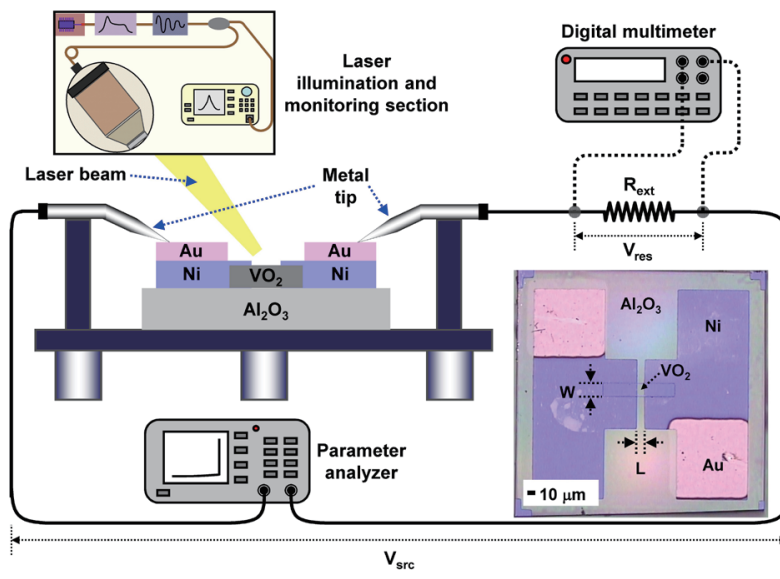

(a)

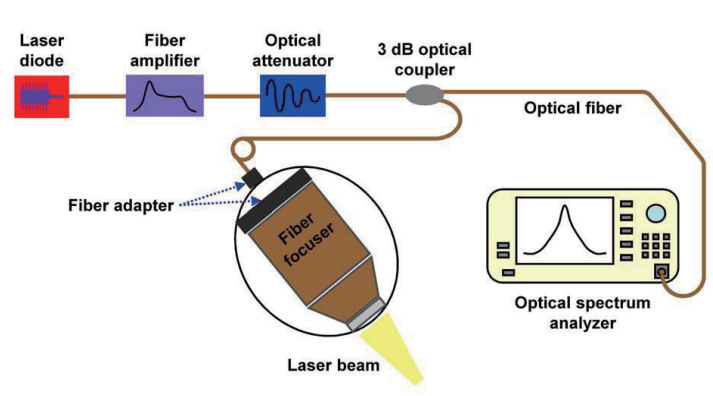

(b)

Fig. 1. (Color online) (a) Experimental setup for $15 \mathrm{~mA}$ laser triggering in $\mathrm{VO}_{2}$-based device and (b) laser illumination and monitoring section. An inset of (a) shows the optical microscopy image of the fabricated device.

$\mathrm{VO}\left(\mathrm{OC}_{3} \mathrm{H}_{7}\right)_{3}$ (Stream Ltd., USA), in isopropanol using acetic acid as a catalyst. The prepared solution was spin-coated onto $\mathrm{Al}_{2} \mathrm{O}_{3}$ substrates at a spin rate of $2000 \mathrm{rpm}$ for $20 \mathrm{~s}$. Then, in order to remove the excess alcohol, the coated film was baked on a hot plate at $250{ }^{\circ} \mathrm{C}$ for $3 \mathrm{~min}$. All the processes were performed in air to partially hydrolyze the alkoxide film with ambient moisture and repeated three times in a row. The final annealing process was carried out at $410{ }^{\circ} \mathrm{C}$ for $30 \mathrm{~min}$ in air. To form the $\mathrm{VO}_{2}$ phase through a reduction process, additional annealing of the film was carried out at a low oxygen pressure. By utilizing a photolithographic technique, $\mathrm{Au} / \mathrm{Ni}$ electrodes were formed on etched $\mathrm{VO}_{2}$ films to fabricate two-terminal microdevices. $L$ and $W$ indicate the electrode separation and the width of the $\mathrm{VO}_{2}$ patch, respectively. In the laser illumination and monitoring section shown in Fig. 1(b), the output of a distributed feedback LD (Thorlabs S3FC1550) was amplified by an optical fiber amplifier (Luxpert LXI-2000). The typical output power and side-mode suppression ratio of the LD were $\sim 1.5 \mathrm{~mW}$ and $>40 \mathrm{~dB}$, respectively, and its output power stability was $\pm 0.1 \mathrm{~dB}$ for $24 \mathrm{~h}$. The amplified optical power was adjusted by an optical attenuator with a dynamic range of $60 \mathrm{~dB}$. The attenuator output was separated into two light components via a $3 \mathrm{~dB}$ optical coupler. ${ }^{(18)}$ One component was introduced into a fiber-pigtailed focuser to focus an incident beam on the $\mathrm{VO}_{2}$ film, and the other component into an optical spectrum analyzer (Yokogawa AQ6370) for spectral monitoring. The beam emerging from the focuser illuminated the film at $30^{\circ}$ incidence. The spot diameter and working distance of the focuser were $\sim 18 \mu \mathrm{m}$ and $\sim 10 \mathrm{~mm}$, respectively, and its position was set using an $x y z$ translation stage for the surface spot diameter to be $\sim 90 \mu \mathrm{m}$. The optical density of the 
beam was $\sim 157.2 \mathrm{~W} / \mathrm{cm}^{2}$ at an input power of $10 \mathrm{~mW}$. For measuring the $I-V$ property of the device, a parameter analyzer (HP 4156C) and a microprobe station were utilized. Transient responses of electrical currents were measured in a closed-loop circuit, shown in Fig. 1(a), constructed by serially connecting a resistor with the resistance $R_{\text {ext }}$, the parameter analyzer as a voltage source for the DC bias $V_{\text {src }}$, and the device, by monitoring the voltage across $R_{\text {ext }}$ using a digital multimeter (Keithley 2000).

\section{Experimental Results and Discussion}

Figure 2(a) shows the photoinduced $I-V$ characteristics of the fabricated $\mathrm{VO}_{2}$-based device $(L=10 \mu \mathrm{m}$ and $W=10 \mu \mathrm{m})$, measured in a current-controlled or I-mode. The optical power of the illumination laser with a wavelength of $\sim 1549.99 \mathrm{~nm}$ was varied from -40 to $11 \mathrm{dBm}$, and the current compliance was set as $2 \mathrm{~mA}$. As can be seen from the figure, upper and lower threshold voltages ( $V_{\text {th1,off }}$ and $\left.V_{\text {th2,off }}\right)$ decrease with increasing illumination power. For example, $V_{\text {th1,off }}$ decreases from $\sim 9.6$ to $\sim 4.1 \mathrm{~V}$ at an illumination power of $11 \mathrm{dBm}$. In particular, the difference between the two threshold voltages $\left(V_{\text {thl,off }}\right.$ and $V_{\text {th2,off }}$ ) also decreases, resulting in the collapse of the NDR region as the illumination power increases. The fabricated devices showed good repeatability in $I-V$ measurements for continuous wave laser illumination $(\sim 1550 \mathrm{~nm})$ up to $\sim 1500 \mathrm{~W} / \mathrm{cm}^{2}$.

Figure 2(b) shows the $I-V$ characteristics of the fabricated device $(L=10 \mu \mathrm{m}$ and $W$ $=30 \mu \mathrm{m})$, measured in the I-mode with the laser switched off or on, indicated as blue squares or red circles, respectively. The current compliance was set as $15 \mathrm{~mA}$, which corresponded to $500 \mathrm{kA} / \mathrm{cm}^{2}$ in the device. The optical power, wavelength, and signalto-noise ratio of the illumination laser were $\sim 11.60 \mathrm{dBm}, \sim 1549.99 \mathrm{~nm}$, and $\sim 56.69 \mathrm{~dB}$, respectively. When the laser was switched off, the upper and lower threshold voltages,

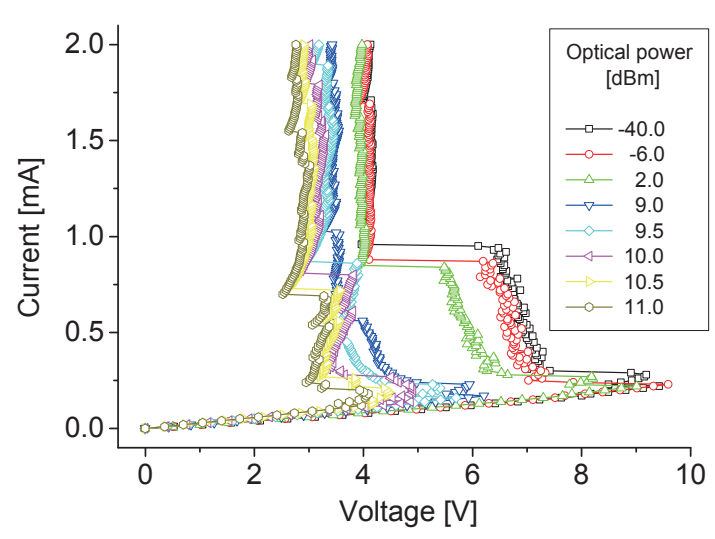

(a)

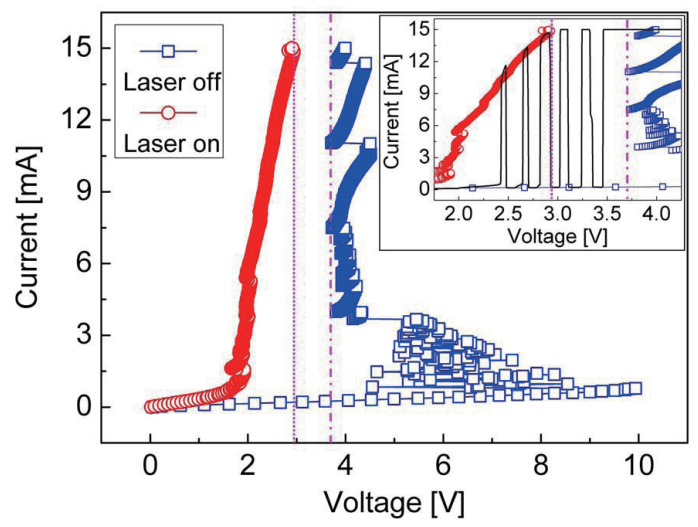

(b)

Fig. 2. (Color online) (a) Photoinduced $I-V$ characteristics of fabricated device $(L=10 \mu \mathrm{m}$ and $W=10 \mu \mathrm{m})$ measured in I-mode and (b) $I-V$ characteristics of fabricated device $(L=10 \mu \mathrm{m}$ and $W=30 \mu \mathrm{m})$ measured in I-mode with laser switched off (blue squares) or on (red circles). 
$V_{\text {thl,off }}$ and $V_{\text {th2,off }}$, of the device were measured as $\sim 9.94$ and $\sim 3.71 \mathrm{~V}$, respectively. Because the LD emits a Gaussian beam, the tolerance limit with respect to the spatial deviation of the beam spot is wider in the transverse direction of $\mathrm{VO}_{2}$ patches than in the longitudinal direction. In the laser excitation of $\mathrm{VO}_{2}$, the portion of $\mathrm{VO}_{2}$ metallic grains increases with the illumination power, resulting in the decrease in threshold voltage. ${ }^{(19)}$ Therefore, if the position and power of the aligned beam are fixed, it is expected that there will be no significant difference in threshold voltage, although the beam profile will change from a Gaussian distribution to a uniform distribution. ${ }^{(7)}$

With the laser switched on, NDR seems to almost disappear, suppressing threshold switching. Although the upper threshold voltage $\left(V_{\text {thl,on }}\right)$ is not definitely determined, the lower threshold voltage $\left(V_{\text {th2,on }}\right)$ can be determined to be $\sim 1.66 \mathrm{~V}$. The voltage at the compliance current $(\sim 2.94 \mathrm{~V})$, indicated as a dotted line, can act as the effective upper threshold voltage $\left(V_{\text {thl,eff,on }}\right)$ in the laser triggering operation. Bidirectional laser triggering can be achieved in a specific $V_{\text {src }}$ range between $V_{\text {th1,eff,on }}$ and $V_{\text {th2,off }}(\sim 2.94$ and $\sim 3.71 \mathrm{~V}$ ), i.e., a voltage region between dotted and dash-dotted lines. We will designate this specific $V_{\text {src }}$ region as Region $\mathrm{S}$ for convenience. On the other hand, unidirectional laser triggering, in which the reverse $\mathrm{PT}$ of $\mathrm{VO}_{2}$ does not occur even when switching the laser off, occurs at $V_{\text {src }}>V_{\text {th2,off- }}$ Thus, $V_{\text {src }}$ was selected as $3.35 \mathrm{~V}$ to induce bidirectional laser triggering.

This can be confirmed from the inset of Fig. 2(b), which shows optically controlled switching characteristics of the $\mathrm{VO}_{2}$ device under $V_{\text {src }}$ increase from 0 to $4 \mathrm{~V}$ with the laser randomly switched on or off six times per state. In the $V_{\text {src }}$ range of $2.0-3.4 \mathrm{~V}$, the first five bidirectional triggering operations are obtained, and at $V_{\text {src }}>3.4 \mathrm{~V}$, the device current that jumps to $15 \mathrm{~mA}$ with the turning on of the laser does not fall after the turning off of the laser, that is, unidirectional triggering operation is observed at the sixth triggering operation. It can be inferred from this optical gating (triggering) result that the bidirectional triggering regime and, in particular, the maximum on-state current of the optically triggered device are directly determined by the $I-V$ behavior of the device, measured in the I-mode with the laser switched on or off. In addition, it is found that an effective bidirectional triggering region is smaller than Region $\mathrm{S}$ owing to the laser heating effect. For example, because the $I-V$ curves in Fig. 2(b) are measured at $\sim 3 \mathrm{~s}$ after switching the continuous wave laser on or off, the $V_{\text {th2,off }}$ of the device that experiences the instantaneous change in illumination power is slightly smaller than the upper limit $(\sim 3.71 \mathrm{~V})$ of Region S.

Figure 3 shows the transient responses of laser-triggered devices for various onstate temporal durations. The compliance current was also set as $15 \mathrm{~mA}$ for all transient responses. Figures 3(a) and 3(b) show the transient responses of the fabricated $\mathrm{VO}_{2}$ device laser-triggered for relatively shorter $(5,10$, and $20 \mathrm{~s})$ and longer $(30,60$, and 120 s) on-state durations, respectively, in the closed-loop circuit with $R_{\text {ext }}=10 \Omega$ and $V_{\text {src }}=$ 3.35 V. In Fig. 3(a), the bidirectional triggering operation was performed five times for each on-state duration. The off-state duration, i.e., the illumination-free interval between two adjacent triggering operations, was set to be the same as the corresponding on-state duration, except for the interval between two triggering operations with different onstate durations. In Fig. 3(b), six bidirectional triggering operations were sequentially 


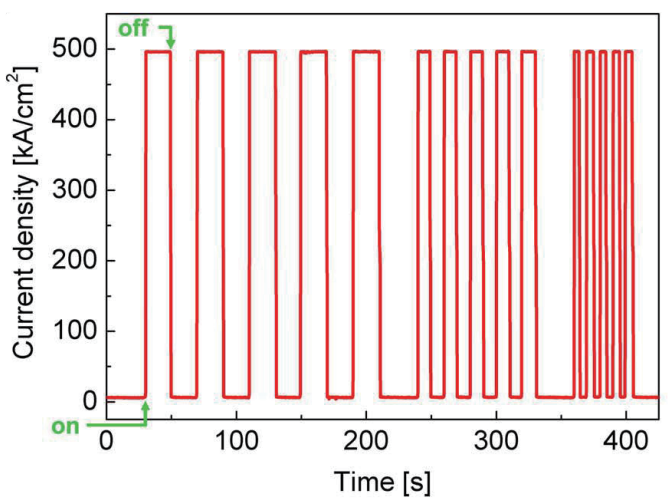

(a)

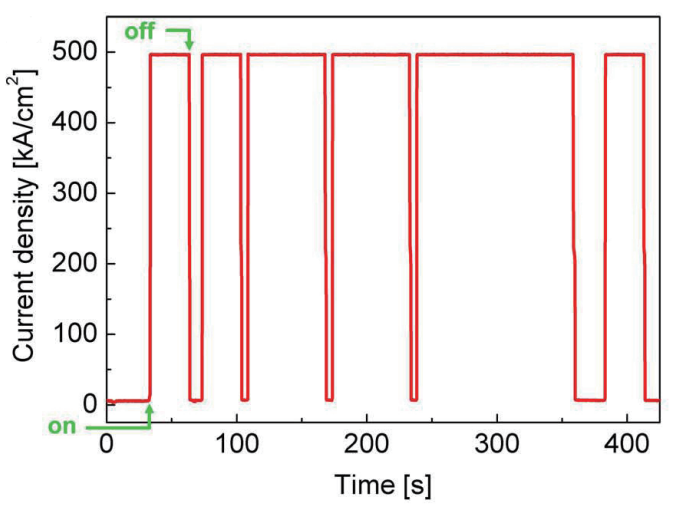

(b)

Fig. 3. (Color online) Transient responses of laser-triggered device for relatively (a) short on-state durations $(5,10$, and $20 \mathrm{~s})$ and (b) long on-state durations $(30,60$, and $120 \mathrm{~s})$. In the closed loop circuit, the $\mathrm{VO}_{2}$ device was biased at $V_{\text {src }}=3.35 \mathrm{~V}$ with $R_{\text {ext }}=10 \Omega$.

performed for on-state durations of $30,30,60,60,120$, and $30 \mathrm{~s}$, and off-state durations, unlike the case in Fig. 3(a), were irregularly selected among 5, 10, and 20 s. It was observed from the measured responses that stable bidirectional switching between $\sim 0.19$ and $15 \mathrm{~mA}$ can be obtained at $V_{\text {src }}=3.35 \mathrm{~V}$ for various on- or off-state durations. The average amplitude switching ratio was evaluated as $\sim 78.9$ in the bidirectional laser triggering of the fabricated device.

In particular, the rising time was measured as $\sim 192 \mathrm{~ms}$ regardless of the onstate duration, which was similar to the minimum data acquisition time step in our experimental setup. The actual rising time is expected to be shorter than the measured one, and the measured response time will decrease if data acquisition is performed at a higher rate. The rising time can also be affected by the long switching time of the laser driving current pulse. The falling time, measured as $\sim 608 \mathrm{~ms}$, was longer than the rising time, but it also showed little dependence on the on-state duration. Slower falling was attributed to the laser heating effect and increased thermal conductivity in the metallic state of $\mathrm{VO}_{2}$. In the laser-induced PT, the thermally induced PT dominates over photoexcitation at an illumination power higher than $30 \mathrm{~mW} \cdot{ }^{(19)}$ Laser-induced heating increases the portion of metallic $\mathrm{VO}_{2}$ grains in the $\mathrm{VO}_{2}$ film and decreases the threshold voltage of the $\mathrm{VO}_{2}$ device while reducing a bidirectional triggering region such as Region S. The reduction of the bidirectional triggering region deteriorates the amplitude switching ratio owing to the increase in off-state current. Here, we focused on the on-state durations, which are on the order of $10 \mathrm{~s}$, to examine the laser-induced heating effect, coupled with the photoinduced PT. Previous studies related to the thermal switching of $\mathrm{VO}_{2}$ reported a switching time of $1-3 \mathrm{~ms},{ }^{(8,9)}$ and the voltage-triggered PT has an ultrashort switching time of $\sim 2$ ns. ${ }^{(15)}$ If another data collection instrument with a high sampling rate is used for shorter on-state durations, it is expected for the actual 
switching time to be on the order of ms. In particular, the response time can be further improved by incorporating high-speed external optical switches to generate short laser pulses or thermoelectric coolers to control the device temperature affected by laserinduced heating. Moreover, the thickness dependence of the device performance should be considered. Generally, the average $\mathrm{VO}_{2}$ grain size increases with the increase in the thickness of the $\mathrm{VO}_{2}$ film. The amplitude and sharpness of the thermal PT of $\mathrm{VO}_{2}$ are directly correlated with the grain size. With the increase in grain size, resulting from the increase in film thickness, the density of grain boundaries and associated defects decreases, causing a stronger and sharper transition. On the other hand, the amplitude of hysteresis curves decreases with the decrease in film thickness. ${ }^{(19)}$ This will lead to the decrease in maximum on-state current and thus amplitude switching ratio.

\section{Conclusions}

We demonstrated bidirectional laser triggering in a two-terminal planar $\mathrm{VO}_{2}$ device using an approximately $1550 \mathrm{~nm}$ LD. A maximum on-state current of $15 \mathrm{~mA}$ and an amplitude switching ratio of $\sim 78.9$ were attained in the $\mathrm{VO}_{2}$ device DC-biased at 3.35 $\mathrm{V}$, and the transient response of light-triggered currents was also analyzed. The high amplitude switching ratio coupled with the bidirectional switching capability could lead to further interest in the use of light-triggered oxide devices for future electronic power devices and sensing systems. ${ }^{(20)}$

\section{Acknowledgements}

This research was supported by the Korea Electric Power Corporation Research Institute through the Korea Electrical Engineering \& Science Research Institute (grant number: R13TA12).

\section{References}

1 F. J. Morin: Phys. Rev. Lett. 3 (1959) 34.

2 E. Arcangeletti, L. Baldassarre, D. D. Castro, S. Lupi, L. Malavasi, C. Marini, A. Perucchi and P. Postorino: Phys. Rev. Lett. 98 (2007) 196406.

3 A. Cavalleri, Cs. Tóth, C. W. Siders, J. A. Squier, F. Ráksi, P. Forget and J. C. Kieffer: Phys. Rev. Lett. 87 (2001) 237401.

4 Y. W. Lee, B.-J. Kim, J.-W. Lim, S. J. Yun, S. Choi, B.-G. Chae, G. Kim and H.-T. Kim: Appl. Phys. Lett. 92 (2008) 162903.

5 Y. W. Lee, B.-J. Kim, S. Choi, H.-T. Kim and G. Kim: Opt. Express 15 (2007) 12108.

6 Y. W. Lee, E.-S. Kim, B.-S. Shin and S.-M. Lee: KIEE J. Electr. Eng. Technol. 7 (2012) 784.

7 B.-J. Kim, G. Seo and Y. W. Lee: Opt. Express 22 (2014) 9016.

8 S. Chen, H. Ma, X. Yi, H. Wang, X. Tao, M. Chen, X. Li and C. Ke: Infrared Phys. Technol. 45 (2004) 239.

9 H. Wang, X. Yi, S. Chen and X. Fua: Sens. Actuators, A 122 (2005) 108.

10 R. M. Briggs, I. M. Pryce and H. A. Atwater: Opt. Express 18 (2010) 11192.

11 J. D. Ryckman, V. Diez-Blanco, J. Nag, R. E. Marvel, B. K. Choi, R. F. Haglund and S. M. Weiss: Opt. Express 20 (2012) 13215. 
12 J. D. Ryckman, K. A. Hallman, R. E. Marvel, R. F. Haglund and S. M. Weiss: Opt. Express 21 (2013) 10753.

13 P. A. Do, A. Hendaoui, E. Mortazy, M. Chaker and A. Hache: Opt. Commun. 288 (2013) 23.

14 B.-G. Chae, H.-T. Kim, D.-H. Youn and K.-Y. Kang: Physica B 369 (2005) 76.

15 Y. Zhou, X. Chen, C. Ko, Z. Yang, C. Mouli and S. Ramanathan: IEEE Electron Dev. Lett. 34 (2013) 220.

16 N.-S. Lee, J.-S. Chang, and Y.-S. Kwon: KIEE J. Electr. Eng. Technol. 1 (2006) 366.

17 B.-G. Chae, H.-T. Kim, S. J. Yun, Y. W. Lee, B.-J. Kim, D.-H. Youn and K.-Y. Kang: Electrochem. Solid-State Lett. 9 (2006) C12.

18 Y.-K Choi: KIEE J. Electr. Eng. Technol. 4 (2009) 287.

19 G. Seo, B.-J. Kim, H.-T. Kim and Y. W. Lee: J. Lightwave Technol. 30 (2012) 2718.

20 B. V. Manikandan, S. C. Raja and P. Venkatesh: KIEE J. Electr. Eng. Technol. 6 (2011) 14. 